\section{International Scientific Journal Theoretical \& Applied Science}

\author{
p-ISSN: 2308-4944 (print) e-ISSN: 2409-0085 (online) \\ Year: $2018 \quad$ Issue: $01 \quad$ Volume: 57 \\ Published: $30.01 .2018 \quad$ http://T-Science.org
}

Sergey Alexandrovich Mishchik

Associate Professor, Candidate of Pedagogical Science, Academician of International Academy TAS, Assistant professor Department of Physics, State Maritime University Admiral Ushakov, Russia, sergei_mishik@mail.ru

SECTION 21. Pedagogy. Psychology. Innovation in Education

\title{
SYSTEMIC PROBLEMS MOVEMENT OF PARTICLES IN A MAGNETIC FIELD OF APPLIED PHYSICS MARITIME FLOT OF PEDAGOGOMETRIC ANALYSIS
}

\begin{abstract}
The basic principles of the system of problems movement of particles in a magnetic field in applied physics Navy pedagogometric analysis of the formation of mathematical models of learning activities about the nature of achieving the criteria of life, cycling, systemsness and phasing, which form a basic cell of the educational space, as well as prima nenie twelve pointed star Ertsgammy relatively presentation ertsgamming principle which determines the foundations pedagogometric through forming substantive methods of hyper-space professional life, psychological and educational activity theory, psycho-pedagogical system analysis and the theory of the formation of mental actions.

Key words: pedagogometric, vital activity, cyclicity, system, phase, star Erzgammy, movement of particles in a magnetic field, applied physics, marine fleet.

Language: Russian

Citation: Mishchik SA (2018) SYSTEMIC PROBLEMS MOVEMENT OF PARTICLES IN A MAGNETIC FIELD OF APPLIED PHYSICS MARITIME FLOT OF PEDAGOGOMETRIC ANALYSIS. ISJ Theoretical \& Applied Science, 01 (57): 145-151.

Soi: http://s-o-i.org/1.1/TAS-01-57-26 Doi: crossef https://dx.doi.org/10.15863/TAS.2018.01.57.26

УДК 372.851

\section{СИСТЕМНЫЕ ЗАДАЧИ ДВИЖЕНИЯ ЧАСТИЦ В МАГНИТНОМ ПОЛЕ} ПРИКЛАДНОЙ ФИЗИКИ МОРСКОГО ФЛОТА ПЕДАГОГОМЕТРИЧЕСКОГО АНАЛИЗА

Аннотация: рассмотрены основные принципы построения системных задач движения частиц 6 магнитном поле прикладной физики морского флота педагогометрического анализа при формировании математических моделей учебной деятельности относительно характера достижения критериев жизнедеятельности, цикличности, системности и этапности, которые образуют базисную ячейку образовательного пространства, а также применение двенадиати конечной звезды Эригаммы относительно представления приниипа эригаммности, который определит основы педагогометрики через формообразование предметными методами гиперпространства профессиональной жизнедеятельности, психолого-педагогической теории деятельности, психолого-педагогического системного анализа и теории формирования умственных действий.

Ключевые слова: педагогометрика, жизнедеятельность, ияиличность, системность, этапность, звезда Эризгаммы, движение частиц в магнитном поле, прикладная физика, морской флот.

\section{Introduction}

Формирование системных задач движения частиц в магнитном поле прикладной физики морского флота педагогометрического анализа гармонизируются с решением общей задачи педагогометрики - - установление математических моделей учебной деятельности через базисный процесс методологии

педагогометрического анализа, выражающего заданную структуру и форму жизнедеятельности, цикличности, системности и этапности. Предложенный педагогометрический анализ устанавливает базисную ячейку образовательного пространства, которая отражает принцип эрцгаммности через всеобщую структуру двенадцати конечной звезды
\end{abstract}


Эрцгаммы. Представленная зависимость определяет основы педагогометрики через выделение предметных методов гиперпространства профессиональной жизнедеятельности, психолого-педагогической теории деятельности, психолого-педагогического системного анализа и теории формирования умственных действий $[1,2,3]$.

Представленные основы подготовки инновационных широкопрофильных специалистов направлены на совершенствование базы предметных прикладных профессиональных задач движения частиц в магнитном поле прикладной физики морского флота педагогометрического анализа, относительно проектной профессиональной деятельности на морском флоте. Проектирование математических моделей учебно-профессиональной деятельности специалистов инновационного мышления ориентируется на: базисную звезду Эрцгаммы гиперпространства жизнедеятельности (Е1); базисный целостно-системный цикл жизнедеятельности (Е2); базисную звезду Эрцгаммы системного анализа (Е3); базисное проявление двенадцати этапов и форм познавательного гиперпространства жизнедеятельности образовательного процесса (E4) [4,5,6].

Представленные системные задачи движения частиц в магнитном поле прикладной физики морского флота педагогометрического анализа и проектная база предметных педагогометрических моделей эрцгаммного анализа образовательных объектов с признаком базисно-нормативной эрцгаммности, определяют их обобщённые структуры. В данном случае устанавливается собственная функция психолого-математического представления профессионально-значимых объектов системных задач движения частиц в магнитном поле прикладной физики морского флота педагогометрического анализа через объединение признаков смыслообразования учебно-профессионального действия, его принятия, ориентировочно-исполнительноконтрольных признаков и прогноза совершенствования анализа объектов педагогометрческого содержания [7,8,9].

\section{Materials and Methods}

Системные задачи движения частиц в магнитном поле прикладной физики морского флота определяют целостно-системное моделирование основных элементов транспортных технических объектов. Это моделирует формирование сознательной ориентации на единство базисных характеристик предметных и исполнительных условий относительно предмета содержания и способа его реализации через представление базисной ячейки образовательного пространства, выражающего принцип эрцгаммности адекватного структуре двенадцати конечной звезды Эрцгаммы. Рассматриваются: радиус траектории окружности, по которой движется электрон в электронно-лучевой трубке судовой навигационной системе, период обращения и момент импульса движения электрона в магнитном поле судовой навигационной системы прикладной физики на морском флоте [10, 11,12].

В процессе решения системных задач движения частиц в магнитном поле прикладной физики морского флота необходимо применять основные положения теории деятельности, системного анализа и теории формирования интеллекта через построение математических моделей учебно-профессиональной активности отражающей структуру: базисной звездой Эрцгаммы гиперпространства жизнедеятельности (Е1); базисного целостно-системного циклом жизнедеятельности (E2); базисной звездой Эрцгаммы системного анализа (Е3); базисного проявления двенадцати этапов и форм познавательного гиперпространства жизнедеятельности относительно образовательного процесса (E4).

Системный анализ предполагает выполнение последовательности системных аналитических действий: выделить объект анализа - задачу движения частиц в магнитном поле прикладной физики морского флота (ЗДЧВМППФМФ) как систему; установить порождающую среду ЗДЧВМППФМФ; определить уровни анализа ЗДЧВМППФМФ; представить целостные свойства ЗДЧВМППФМФ относительно пространственных, и временных характеристик и их комбинаций; выделить структуру уровня анализа ЗДЧВМППФМФ; установить структурные элементы уровня анализа ЗДЧВМППФМФ; определить системообразующие связи данного уровня анализа ЗДЧВМППФМФ; представить межуровневые связи анализа ЗДЧВМППФМФ; выделить форму организации ЗДЧВМППФМФ; установить системные свойства и поведение ЗДЧВМППФМФ.

\section{Задача 1}

В электронно-лучевой трубке судовой навигационной системы электрон, ускоренный разностью потенциалов $\boldsymbol{U}=\boldsymbol{l} \boldsymbol{\kappa} \boldsymbol{B}$, влетает в однородное магнитное поле, направление которого перпендикулярно к направлению его движения. Индукция магнитного поля $\boldsymbol{B}=1,19$

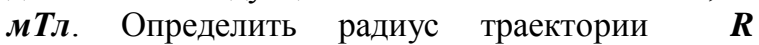
окружности, по которой движется электрон в электронно-лучевой трубке судовой 
навигационной системе, период обращения $\boldsymbol{T}$ и момент импульса $\boldsymbol{M}$ электрона. $\kappa 2 \cdot M^{2} / c$.

Ответ: $R=9$ cм; $T=30$ нс $; M=1,5 \cdot 10^{-24}$

\section{Задача 2}

В судовой электромагнитной системе электрон, ускоренный разностью потенциалов $\boldsymbol{U}$ $=300 \boldsymbol{B}$, движется параллельно прямолинейному длинному индукционному проводу на расстоянии $\boldsymbol{a}=4 \boldsymbol{м} \boldsymbol{м}$ от него. Определить силу $\mathrm{F}$, действующую на индикаторный электрон судовой электромагнитной системы, если по индукционному проводнику следует сила тока $I=5 \mathrm{~A}$.

$$
\text { Ответ: } F=4 \cdot 10^{-16} \mathrm{H} \text {. }
$$

\section{Задача 3}

В электронно-лучевой трубке судовой навигационной системе электрон влетает в однородное магнитное поле, направление которого перпендикулярно к направлению его движения. Скорость электрона $\boldsymbol{v}=4 \cdot 10^{7} \boldsymbol{m} / \boldsymbol{c}$. Индукция магнитного поля $\boldsymbol{B}=\boldsymbol{1} \boldsymbol{м} \boldsymbol{T}$. Определить тангенциальное $\boldsymbol{a}_{\boldsymbol{\tau}}$ и нормальное $\boldsymbol{a}_{\boldsymbol{n}}$ ускорения электрона в магнитном поле электронно-лучевой трубки судовой навигационной системы.

\section{Ответ: $a_{\tau}=0$ во все время движения; $a_{n}=$ const $=7 \cdot 10^{15} \mathrm{M} / \mathrm{c}^{2}$.}

\section{Задача 4}

Для протона, движущегося в судовом электромагнитном индикаторе по дуге окружности радиусом $\boldsymbol{R}=\boldsymbol{6 0} \boldsymbol{c m}$ в магнитном поле с индукцией $\boldsymbol{B}=\boldsymbol{I} \boldsymbol{T}$, определить кинетическую энергию $\boldsymbol{W}$ (в электрон-Вольтах)

Ответ: $W=17,3 \mathrm{MэB}$.

\section{Задача 5}

В электронно-лучевой трубке судовой навигационной системы пучок электронов, ускоренных разностью потенциалов $\boldsymbol{U}=300 \boldsymbol{B}$, влетает в однородное магнитное поле, направленное от чертежа к нам. Ширина поля $\boldsymbol{b}=\mathbf{2 , 5} \boldsymbol{c m}$. В отсутствие магнитного поля пучок электронов дает пятно в точке $\boldsymbol{A}$ флуоресцирующего экрана, расположенного на расстоянии $\boldsymbol{\ell}=\mathbf{5} \boldsymbol{c \boldsymbol { M }}$ от края полюсов магнита. При включении магнитного поля пятно смещается в точку $\boldsymbol{B}$. Определить смещение $\boldsymbol{x}=\boldsymbol{A B}$ пучка электронов в электронно-лучевой трубке судовой навигационной системы, индукция магнитного поля $B=14,6 . м \kappa T л$.

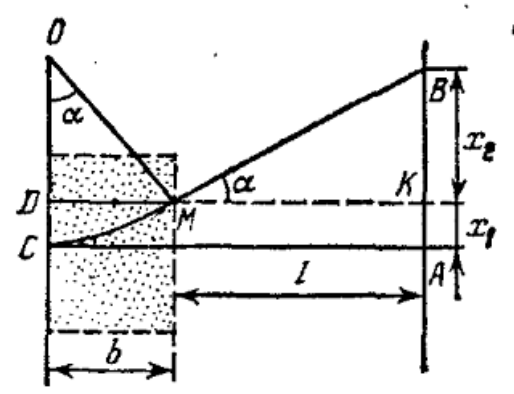

Рисунок 1 - Схема траектории электронов.

Ответ: $R=4$ cм и $x=4,9$ cм.

\section{Задача 6}

В электронно-лучевой трубке судовой навигационной системы электрон, ускоренный разностью потенциалов $\boldsymbol{U}=\boldsymbol{6} \boldsymbol{\kappa} \boldsymbol{B}$, влетает в однородное магнитное поле под углом $\alpha=30^{\circ}$ к направлению поля и движется по винтовой траекторий. Индукция магнитного поля $\boldsymbol{B}=13$ $\boldsymbol{M} \boldsymbol{T} \boldsymbol{л}$. Определить радиус $\boldsymbol{R}$ и шаг $\boldsymbol{h}$ винтовой траектории электрона в электронно-лучевой трубке судовой навигационной системы.

Ответ: $R=1 \mathrm{~cm}$ и $h=11 \mathrm{~cm}$.

\section{Задача 7}

В судовой электромагнитной системе электрон влетает в плоский горизонтальный конденсатор параллельно его пластинам со скоростью $\boldsymbol{v}=\mathbf{1 0}^{7} \boldsymbol{M} / \boldsymbol{c}$. Длина конденсатора $\boldsymbol{\ell}=\mathbf{5}$ c.M. Напряженность электрического поля конденсатора $\boldsymbol{E}=\mathbf{1 0} \boldsymbol{\kappa} \boldsymbol{B} / \boldsymbol{M}$. При вылете из конденсатора электрон попадает в магнитное поле судовой электромагнитной системы, перпендикулярное к электрическому полю.

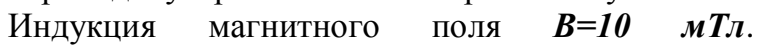
Определить радиус $\boldsymbol{R}$ и шаг $\boldsymbol{h}$ винтовой траектории электрона в магнитном поле судовой электромагнитной системы.

Ответ: $R=5$ мм; $h=3,6$ см .

\section{Задача 8}

В судовой электромагнитной системе электрон, ускоренный разностью потенциалов $\boldsymbol{U}$ $=3 \boldsymbol{\kappa} \boldsymbol{B}$, влетает в магнитное поле соленоида под углом $\boldsymbol{\alpha}=30^{\circ}$ к его оси. Число Ампер-витков соленоида $\boldsymbol{I N}=\mathbf{5 0 0 0} \boldsymbol{A} \cdot \boldsymbol{\theta}$. Длина соленоида $\boldsymbol{\ell}=\mathbf{2 5}$ $\boldsymbol{c m}$. Найти шаг $\boldsymbol{h}$ винтовой траектории электрона в магнитном поле судовой электромагнитной системе.

Ответ: $h=3,94$ см . 


\begin{tabular}{|c|c|c|c|c|c|c|}
\hline Impact Factor: & $\begin{array}{l}\text { ISRA (India) } \\
\text { ISI (Dubai, UAE } \\
\text { GIF (Australia) } \\
\text { JIF }\end{array}$ & $\begin{array}{l}=1.344 \\
=0.829 \\
=0.564 \\
=1.500\end{array}$ & $\begin{array}{l}\text { SIS (USA) } \\
\text { PИНЦ (Russia) } \\
\text { ESJI (KZ) } \\
\text { SJIF (Morocco) }\end{array}$ & $\begin{array}{l}=0.912 \\
=0.207 \\
=4.102 \\
=2.031\end{array}$ & $\begin{array}{l}\text { ICV (Poland) } \\
\text { PIF (India) } \\
\text { IBI (India) }\end{array}$ & $\begin{array}{l}=6.630 \\
=1.940 \\
=4.260\end{array}$ \\
\hline
\end{tabular}

\section{Задача 9}

В судовой электромагнитной системе магнитное поле напряженностью $\boldsymbol{H}=\boldsymbol{8} \boldsymbol{\kappa} \boldsymbol{A} / \boldsymbol{M}$ и электрическое поле напряженностью $\boldsymbol{E}=\boldsymbol{1} \boldsymbol{\kappa} \boldsymbol{B} / \boldsymbol{M}$ направлены одинаково. Электрон влетает в электромагнитное поле со скоростью $\boldsymbol{v}=10^{5} \mathrm{~m} / \boldsymbol{c}$. Найти нормальное $\boldsymbol{a}_{\boldsymbol{n}}$, тангенциальное $\boldsymbol{a}_{\boldsymbol{\tau}}$ и полное $\boldsymbol{a}$ ускорения электрона в судовой электромагнитной системе. Задачу решить, если скорость электрона направлена: а) параллельно направлению электрического поля; б) перпендикулярно к направлению электрического поля судовой электромагнитной системы.

Ответ:

a) $a_{n}=0 ; a=a_{\tau}=e E / m=1,76 \cdot 10^{14} \mathrm{M} / \mathrm{c}^{2}$;
b) $a_{\tau}=0 ; a=a_{n}=2,5 \cdot 10^{14} \mathrm{M} / \mathrm{c}^{2}$

\section{Задача 10}

Протон влетает в однородное магнитное поле судовой электромагнитной системы под углом $\alpha=30^{\circ}$ к направлению поля и движется по винтовой линии радиусом $R=1,5 \boldsymbol{c m}$. Индукция магнитного поля $\boldsymbol{B}=\mathbf{0 , 1} \boldsymbol{T}_{\boldsymbol{\pi}}$. Определить кинетическую энергию $\boldsymbol{W}$ протона в однородном магнитном поле судовой электромагнитной системы.

\section{Ответ: $W=433$ эB.}

\section{Conclusion}

Основные направления формирования и развития инновационного широкопрофильного профессионального мышления связываются с организацией всестороннего развития педагогометрической эрцгаммности. Предложенные системные задачи движения частиц в магнитном поле прикладной физики морского флота педагогометрического анализа выделяют основные направления развития и совершенствования базы прикладных предметных педагогометрических моделей образовательных объектов относительно педагогометрического математического моделирования учебного процесса. Формируемая образовательная деятельность связывается с процессами совершенствования программируемых математических моделей учебной активности относительно характера достижения критериев жизнедеятельности, цикличности, системности и этапности $[13,14$, 15].

Выделенные содержательные задачи педагогометрического анализа по движению частиц в магнитном поле ориентируются на выработку целостно-системного цикла жизнедеятельности, отражающего последовательность выполняемых фазовых состояний реализации данных учебного процесса, характеризующего исследуемые образовательные явления и представленных во времени в форме различных математических моделей.

Формирование целостно-системного цикла жизнедеятельности есть многоэтапный процесс. Преобразование внешнего образа мира во внутренний происходит в результате постепенной различно функциональной деятельности, которая отражает базисные рефлекторно-физиологические этапы процесса интериоризации относительно общего процесса познания. Педагогометрический анализ данных процессов позволит проводит плановое моделирование условиями подготовки широкопрофильных специалистов и управление формированием профессиональных качеств личности.

Определение в качестве базисного этапа формирования целостно-системной внутренней деятельности процесса ориентации, отражает предметный смысл всей психологопедагогической науки относительно подготовки современных целостно-системных широкопрофильных специалистов, имеющих высший уровень целостно-системной ориентировки в социально-экономических, технических и естественных системах.

Реализация поставленных педагогометрических задач происходит через последовательность системных педагогометрических действий. Психологопедагогический системный анализ занимает базисную позицию в формировании целостносистемной личности и её профессионального образа - специалиста широкого профиля.

Психолого-педагогометрический системный анализ является одним из базисных средств процесса формирования специалиста широкого профиля - профессионального образа целостносистемной личности. Множество действий системного анализа определяют общую технологию решения выделенной проблемы. Важным действием психологопедагогометрического системного анализа является процесс установления по определению целостных свойств системы, формирующих аналитическую фазу процесса исследования.

Целостные свойства системы устанавливают характер сформированных внешних характеристик системы без учёта её сложности, упорядоченности и разнообразия параметров объекта. Эти свойства системы задают характер сформированности относительно цели её развития. Известный тезис, что система в своём развитии стремится к целостности, определяют всё палитру психолого-педагогических условий этой многообразной жизнедеятельности, которая 
отражает четыре основных направления анализа данного процесса.

Первое направление педагогометрического анализа устанавливает собственную структуру данного действия. То есть раскрываются психолого-педагогические направления ориентирующего компонента деятельности по определению целостных свойств объекта. При анализе материальных объектов сразу раскрывается пять основных целостных свойств: пространственные, временные, гравита-ционные, силовые и энергетические. При исследовании широкопрофильности профессиональной деятельности анализируются дополнительно деятельностные формы целостно-системного учебного компонента. Устойчивость целостносистемного учебного процесса определяется уровнем внутренней системности всего объекта и целостно-системного цикла в целом.

Второе направление анализа действия педагогометрического системного анализа по определению целостных свойств объекта характеризуется способом реализации этого процесса. То есть каждая операция целостности характеризуется применением всего анализа по реализации выделенной цели. Применение различных операционных схем исполнения определяет уровень сформированности каждой операции. Выделенная вариативность операций исполнительной части действия позволяет задать решение педагогометрических задач относительно минимаксной или максоминной степени реализации поставленной цели учебнопрофессиональной деятельности.

Углублённое направление анализа действия педагогометрического системного анализа по определению целостных свойств объекта представляется уровнем мониторинга всего процесса достижения выделенной целостности. Можно выделить многочисленную группу таких показателей, которые задаются по характеру целостности самого учебного процесса, каждого элемента цикла, уровнем взаимосвя-зей самих циклов, образующих многомерные кванты взаимодействий учитель-ученик. Характер автоматизации мониторинга устанавливает общий уровень сформированности действия системного анализа по определению целостных свойств системы.

Представленное направление анализа действия системного анализа по установлению целостных свойств объекта определяет уровень интеграции и дифференциации основных параметров целостности. Можно составить базисные многомерные матрицы, которые будут образовывать педагогометрические математические модели учебного процесса. Если одномерная матрица образует элементарный вектор базисности, то двумерная матрица позволяет спроектировать сложную учебнопрофессиональную широкопрофильную модель. Трёхмерная матрица целостно-системного учебного процесса образует гиперпространство учебно-пространственных взаимодействий, которые могут создавать различные облачные образовательные технологии.

По назначению, выделенное действие психолого-педагометрического анализа определить целостные свойства системы, может выполнять три дополнительные фундаментальные функции. Данное педагогометрическое действие задаёт тип учебных теоретических задач, решение которых устанавливает способ раскрытия предмета изучения. Именно целостные свойства объекта раскрывают его многогранные будущие параметры и характеристики, задают уровень нормативной творческой деятельности, когда можно составить компоненты вектора творческой деятельности и сформулировать класс педагогометрических задач, задающих уровень широкопрофильности профессиональной деятельности. Это позволит проектировать соответствующий уровень целостно-системной личности.

Применение действия системного педагогометрического анализа по установлению целостных свойств объекта раскрывает громадные перспективы в области организации лабораторного практикума. В этом случае тематика лабораторных работ позволит создавать циклы лабораторных работ по исследованию и проектированию новых пространственновременных-гравитационно-силовых-

энергетических отношений в многомерных пространствах. Деятельностные компоненты данного процесса по установлению целостных свойств учебно-профессиональной деятельности позволять создавать многовариантные технологии повышенной эффективности исследовательской деятельности от выполнения отдельных практических действий, до создания автоматизированных лабораторных систем.

Максимальная базисно-фундаментальноширокопрофильная эффективность действия системного педагогометрического анализа по определению целостных свойств системы может быть достигнута при решении исследовательских задач различной направленности от учебной до истинно-исследовательской. В этом случае педагогометрический процесс выделения целостных свойств превращается в настоящий исследовательский процесс сформированный не на эмпирических, а теоретических основаниях. Когда чётко выделяются все этапы целостносистемного цикла жизнедеятельности со всеми его двенадцатью компонентами, а также все двенадцать форм развития самого системного 
действия системного анализа по выделению целостных свойств системы многомерного целостно-системного Мира, направленного на воспитание целостно-системной личности относительно формирования основных форм педагогометрического знания: мотивационной, ориентационной, материальной, внешнеречевой, вербально-знаковой и их дополнительных шести форм, которые отражают общий механизм существования не просто метода учебно- профессионального развития, а выражают генезис развития смысла - генеральной линии экспозиции развития целостно-системной широкопрофильной личности, спроектированной педагогометрическими функциями математического моделирования при реализации международных образовательных стандартов морского образования эревнометрической формы алигорамного содержания.

\section{References:}

1. Mishchik SA (2014) Pedagogometrika and mathematical modeling educational activity. Materialy Mezhdunarodnoy nauchnoy konferenctsii "Modern mathematics in science" - 30.06.2014. ISJ Theoretical \&Applied Science 6(14): 54-56 Caracas, Venezuela. doi: http://dx.doi.org/10.15863/TAS.2014.06.14.10

2. Mishchik SA (2014) Simulation training activity methods of mathematical logic. Materialy Mezhdunarodnoy nauchnoy konferenctsii "Eurapean Science and Education" - 30.07.2014. ISJ Theoretical \&Applied Science 6(15): 72-74 Marseille, France. doi: http://dx.doi.org/10.15863/TAS.2014.07.15.13

3. Mishchik SA (2014) Mathematical modeling system integrity-cycle of life activity - first goal pedagogometriki. Materialy Mezhdunarodnoy nauchnoy konferenctsii "European Applied Sciences" - 30.08.2014. ISJ Theoretical \&Applied Science 7(16): 77-79. Aix-en-Provence, France. doi: http://dx.doi.org/10.15863/TAS.2014.08.16.13

4. Mishchik SA (2014) Mathematical modeling system integrity-curricular activities - the second problem pedagogometriki. Materialy Mezhdunarodnoy nauchnoy konferenctsii "European Innovation" - 30.09.2014. ISJ Theoretical \&Applied Science 9(17): 126-128 Martigues, France. doi: http://dx.doi.org/10.15863/TAS.2014.09.17.21

5. Mishchik SA (2014) Mathematical modeling holistic-systemic communicative activity - the third task pedagogometriki. Materialy Mezhdunarodnoy nauchnoy konferenctsii "European Scientific Achievements" 30.10.2014. ISJ Theoretical \&Applied Science 10(18): 45-47 Brighton, UK. doi: http://dx.doi.org/10.15863/TAS.2014.10.18.11
6. Mishchik SA (2014) Mathematical modeling integrity - system performance subject - fourth task pedagogometriki. Materialy Mezhdunarodnoy nauchnoy konferenctsii "Eurapean Science and Technology" 30.11.2014. ISJ Theoretical \&Applied Science 11(19): 51-54 Southampton, UK. doi: http://dx.doi.org/10.15863/TAS.2014.11.19.10

7. Mishchik SA (2015) Pedagogometrik - science and academic subject. Materialy Mezhdunarodnoy nauchnoy konferenctsii "European Technology in Science" 28.02.2015. ISJ Theoretical \& Applied Science 02 (22): 103-106 Malmö, Sweden. doi: http://dx.doi.org/10.15863/TAS.2015.02.22.17

8. Irodov I. E. (1979) Zadachi po obshhej fizike. M.: Nauka, 1979 - p.368.

9. Tokmazov GV (2014) Matematicheskoe modelirovanie $\mathrm{v}$ uchebno-professional'noy deyatel'nosti. Materialy Mezhdunarodnoy nauchnoy konferentsii «Modern mathematics in science» - 30.06.2014. ISJ Theoretical \& Applied Science 6(14): 44-46. - Caracas, Venezuela. doi: http://dx.doi.org/10.15863/TAS.2014.06.14.8

10. Tokmazov GV (2014) Mathematical modeling research skills in educational activity methods of probability theory. Materialy Mezhdunarodnoy nauchnoy konferenctsii "Eurapean Science and Technology" 30.11.2014. ISJ Theoretical \&Applied Science 11(20): 66-69 Southampton, United Kingdom. doi:

http://dx.doi.org/10.15863/TAS.2014.11.19.13

11. Mishhik N.A. (2016) Pravovy'e osnovy' francuzskoj si-stemy' bor'by' s zagryazneniem morya / Nauchny'e issledovaniya: Informaciya, analiz, prognoz [Tekst]: monografiya / [V.E'.Lebedev, A.A.Sviridenko, V.M.Sokolinskij i dr.]; pod obshhej red. prof. 
\begin{tabular}{l|lr|ll|ll} 
& ISRA (India) & $=\mathbf{1 . 3 4 4}$ & SIS (USA) & $=\mathbf{0 . 9 1 2}$ & ICV (Poland) & $=\mathbf{6 . 6 3 0}$ \\
Impact Factor: & ISI (Dubai, UAE) $=\mathbf{0 . 8 2 9}$ & PUHL (Russia) $=\mathbf{0 . 2 0 7}$ & PIF (India) & $=\mathbf{1 . 9 4 0}$ \\
& GIF (Australia) & $=\mathbf{0 . 5 6 4}$ & ESJI (KZ) & $=4.102$ & IIBI (India) & $=\mathbf{4 . 2 6 0}$ \\
& JIF & $=\mathbf{1 . 5 0 0}$ & SJIF (Morocco) & $=\mathbf{2 . 0 3 1}$ & & \\
\hline
\end{tabular}

O.I.Kirikova - Kniga 51.- Voronezh-Moskva, 2016.

12. Mishchik NA (2014) The practice of french justice article 228 of the UN convention on the law of the sea. Materialy Mezhdunarodnoy nauchnoy konferenctsii "The European Science and Education"- 30.07.2014. ISJ Theoretical \& Applied Science 07 (15): 93-97. - Marseille, France.

doi:

http://dx.doi.org/10.15863/TAS.2014.07.15.19

13. Mishhik N.A., Antonenko G.A. (2013) Liniya gorizonta kak gradientny'j perepad $\mathrm{V}$ fotograficheskix izmereniyax dlya celej morexodnoj astronomii//E'kspluataciya morskogo transporta. 2013. № 2 (72). Novorossijsk, p. 23-28.

14. Mishhik N.A. (2000) Optimizaciya metodov morexodnoj astronomii [Tekst]: avto-ref.dis. ... kand. tex. nauk: 05.22.16 / N.A.Mishhik. Novorossijsk, 2000. -24 p.

15. Mishhik N.A. (2000) Optimizaciya metodov morexodnoj astronomii [Tekst]: dis. ... kand. tex. nauk: 05.22.16 / N.A.Mishhik. Novorossijsk, 2000. -188 p. 